\title{
Improving the bowel habits of elderly residents in a nursing home using probiotic
}

\section{fermented milk}

\author{
M. van den Nieuwboer ${ }^{1 *}$, A. Klomp-Hogeterp ${ }^{2}$, S. Verdoorn ${ }^{2}$, L. Metsemakers-Brameijer ${ }^{2}$, T.M. Vriend ${ }^{3}$, E. Claassen ${ }^{1,4}$ \\ and O.F.A. Larsen ${ }^{5}$ \\ ${ }^{1}$ VU University Amsterdam, Athena Institute, De Boelelaan 1085, 1081 HV Amsterdam, the Netherlands; ${ }^{2}$ Talma Hof, \\ Genemuidenstraat 4, 8304 GC Emmeloord, the Netherlands; ${ }^{3}$ Hogeschool van Amsterdam, HvA Bewegen, Sport en Voeding, \\ Dr. Meurerlaan 8, 1067 SM Amsterdam, the Netherlands; ${ }^{4}$ Erasmus Medical Center, Department of Viroscience, P.O. Box \\ 2040, 3000 CA Rotterdam, the Netherlands; ${ }^{5}$ Yakult Nederland BV, Handelsweg 59H, 1181 ZA Amstelveen, the Netherlands; \\ m.vanden.nieuwboer@vu.nl
}

Received: 11 August 2014 / Accepted: 16 November 2014

(c) 2015 Wageningen Academic Publishers

RESEARCH ARTICLE

\begin{abstract}
Our aim was to determine whether a fermented milk drink containing probiotics could improve the bowel habits of frail elderly individuals living in a nursing home. A total of 135 participants were enrolled in this pilot study. The bowel habits (stool quality and bowel movements) were recorded by nursing staff during a baseline period of 3 weeks. After this period participants received daily a fermented milk drink containing minimally $6.5 \times 10^{9}$ colony forming units of Lactobacillus casei Shirota $(L C S)$ for 6 weeks. During this period, bowel habits were recorded and compared to baseline period. Forty-four participants (74-99 years old) were compliant and used for analysis. Consumption of fermented milk containing $L c S$ significantly increased the percentage of ideal stool types per week $(P<0.01)$, lowered the percentage of constipation stool types per week $(P<0.01)$ and significantly lowered the percentage of diarrhoea stool types per week $(P=0.016)$ as compared to the baseline period. The study product had no significant effect on bowel movements. During the study, no changes in laxative usage or adverse events associated with the study product were reported. Our results suggest that a fermented milk containing $L c S$ significantly improves the bowel habits of frail elderly residents in a nursing home. These promising results should be further substantiated by a confirmatory study.
\end{abstract}

Keywords: probiotics, elderly, constipation, diarrhoea, bowel habits

\section{Introduction}

The general population is rapidly ageing due to socioeconomic and medicinal advances. It is estimated that in the near future, the population of children under 5 years will be outnumbered by elderly over 65 years old (Duncan and Flint, 2013). This global issue puts an overwhelming burden on healthcare systems in order to maintain a good quality of life (QoL) for elderly individuals (Duncan and Flint, 2013). Elderly ( $\geq 65$ y) often suffer from more than one chronic condition, and approximately $87 \%$ of elderly individuals living at home receive at least one prescription drug (Barat et al., 2000).
It is well understood that ageing is associated with a change in the microbiota (Biagi et al., 2012). Ageing may cause a change in the Firmicutes to Bacteroidetes ratio (Mariat et al., 2009). Also, there seems to be a decline in the heterogeneity of microbial species with, for example, bifidobacteria becoming less abundant (Biagi et al., 2012). Moreover, the microbiota of individuals in long stay residential care appeared to be less diverse when compared to communitydwelling elderly (Claesson et al., 2012). A clear consensus on the changes of the microbiota upon ageing remains however to be established (Magrone and Jirillo, 2013), and also seems to be complicated by differences caused by geographical origin (Mueller et al., 2006). A disbalance in the microbiota is associated with a variety of illnesses 
(Sekirov et al., 2010). For instance, there are indications that constipation may be associated with changes in the intestinal flora (Van Tongeren et al., 2005). Constipation is a disorder that is especially prevalent amongst the older population (Gandell et al., 2013). The prevalence in nursing homes seems to be even higher with respect to communitydwelling elderly. An American study estimated that the prevalence of constipation may be as high as 74\% in nursing homes (Rao and Go, 2010). In addition to the altered microbiota, certain disease states such as hypothyroidism, hypercalcemia, stroke and Parkinson disease as well as drugs commonly taken by elderly (e.g. antidiarrheal agents, calcium-channel blockers, oral iron supplements and opioid analgesics) are known causes for constipation (Gandell et $a l ., 2013)$. Constipation is associated with a wide variety of complications, especially in the frail elderly population. It often leads to anorexia, nausea and pain associated with functional decline (Gandell et al., 2013). Furthermore, it can lead to a decreased QoL and increased costs for health care facilities to manage the problem.

Restoring the disbalanced microbiota to reduce disease risk by using probiotics might improve the overall health of elderly living in nursing homes. Probiotics are defined by the Food and Agricultural Organization and World Health Organization (FAO/WHO) as live microorganisms that, when administered in adequate amounts, confer a health benefit on the host' (FAO/WHO, 2001); this consensus is recently reinforced by the International Scientific Association for Probiotics and Prebiotics (ISAPP; Hill et al., 2014). Several studies indeed demonstrate a beneficial potential of probiotics for constipation (Chmielewska and Szajewska, 2010; Koebnick et al., 2003). Hence, probiotic intervention amongst the residents may lead to an improvement of the QoL, due to a lowering of the constipation prevalence.

Antibiotic usage, which is relatively high in elderly, disturbs the gastrointestinal (GI) microbiota and may increase the incidence of a Clostridium difficile infection (Hell et al., 2013). This highly prevalent pathogen in the elderly, with a carriage rate of around $20 \%$ in home care residents (Karlström et al., 1998), can cause antibiotic-associated diarrhoea (AAD). Meta-analyses indicate that probiotics may be beneficial for the treatment of AAD (Hell et al., 2013; Hempel et al., 2012; Videlock and Cremonini, 2012). In addition, probiotics for non-AAD, which is also frequently encountered within the elderly population (Van Dessel et al., 2005), show promising results (Floch et al., 2011). Iatrogenic bowel irregularities, such as AAD and $C$. difficile-associated diarrhoea can lead to serious complications. Recent studies, however, demonstrate that administration of a commercially available probiotic leads to substantial healthcare cost-savings (Claassen, 2014).
Multiple studies focusing on management of diarrhoea or constipation using probiotics have appeared over the last years. However, to the best of our knowledge, no research is available that focuses on improving bowel habits (including both diarrhoea and constipation) of residents in nursing homes with a very high age. This pilot study therefore investigates the possibility to improve bowel habits using a fermented milk drink containing Lactobacillus casei Shirota $(L c S)$ on frail elderly residents of a nursing home in the Netherlands.

\section{Methodology}

\section{Design and outcome parameters}

This food supplement pilot study was conducted in a nursing home in the Netherlands between March and May 2013. The study was designed in two phases, a baseline period of 3 weeks (weeks 1-3) and an intervention period of 6 weeks (weeks 4-9; total of 63 days; Supplementary Figure S1). During the baseline period participants did not receive the study product. During the intervention period, participants received daily a fermented milk beverage.

The study product was a commercially available probiotic product, Yakult Original (YOR), containing a minimum of $1.0 \times 10^{8} \mathrm{cfu} / \mathrm{ml}$ L. casei strain Shirota (YIT9029) per bottle. Participants orally received one bottle of $65 \mathrm{ml}$ during breakfast per day (minimally $6.5 \times 10^{9} \mathrm{cfu} /$ day). The study product was provided by Yakult Nederland BV (Amstelveen, the Netherlands).

During the complete course of 9 weeks, participants were accompanied to the bathroom by nursing staff to document the primary outcome of stool quality and bowel movements. Stool quality was determined according to the 7-point Bristol stool scale (developed by K.W. Heaton and S.J. Lewis, Bristol University). Bristol stool type 1 and 2 were associated with constipation, type 3 and 4 with ideal stool and type 5, 6 and 7 with diarrhoea (Supplementary Figure S2). Bowel movements were calculated as the number of stools per time-unit and related to the Rome III criteria for functional constipation (Drossman et al., 2006). The criterion for functional constipation was $\leq 2$ stools per week. In addition to bowel habits, other parameters, such as laxative usage and adverse events were monitored.

\section{Study population and criteria}

The study population consisted of 'high maintenance' frail elderly individuals, with a limited life-span (average $\sim 1.5$ year), living in a nursing home. All participants were recruited from the same nursing home after informed consent was obtained from first degree relatives. Residents in the nursing home ageing $\geq 70$ years were considered eligible for inclusion. Participants were excluded from 
the study if (1) their life expectancy was $\leq 6$ months; (2) required intensive medication; or (3) were severely ill (as determined by the in-house physician).

Subjects were accompanied to the bathroom by nursing staff. This also allowed for consistent and reliable data collection. In addition, during the baseline period of 3 weeks, subjects were allowed to consume a maximum of two bottles of YOR. During the intervention period, subjects were allowed to miss a maximum of two bottles of YOR. During the complete course of the study, participants were not allowed to consume any other probiotic products. There were no major alterations in regular diet of the participants during the study as controlled by the institution.

\section{Statistical analysis}

The sample size was estimated based on data provided by Koebnick et al. (2003). Taking into account poor compliance and the sequential design of the study, a sample size of 135 participants would provide sufficient power for this pilot study. All data were tested for normal distribution using a Shapiro-Wilk test in combination with Q-Q (QuantileQuantile) plots for additional verification. Normally distributed data were analysed using a paired t-test, whereas not normally distributed data were analysed using a Wilcoxon-signed rank test (a non-parametric test). Data analysis was performed using R Studio (version 0.98.501) statistical software, and IBM SPSS statistics v.20 (IBM, Armonk, NY, USA). A $P$-value $<0.05$ was considered significant.

\section{Results}

In total 135 subjects gave consent and were recruited to participate in the study. Table 1 describes the baseline characteristics of the study population. Due to serious noncompliance (subjects went to the bathroom independently), only 44 subjects with all data on bowel habits available were considered eligible for analysis. The age range of the perprotocol (PP) population was 74-99 years old (mean $83.8 \mathrm{y}$ ).

There was no difference in laxative usage; the average number of laxatives used per patient per week during intervention was not significantly different from the baseline period $(P=0.091)$. Seven subjects $(15.9 \%)$ did not receive laxatives during the entire study duration. All laxatives were empirically supplemented, with Movicolon as the most frequent prescribed laxative. Other laxatives were lactulose, plum compote, bisacodyl, Norgalax and magnesium-oxide $(\mathrm{MgO})$.

The total number of stools per week per participant during the baseline period was $5.2 \pm 1.5$ compared to $5.4 \pm 1.7$ stools per week during the intervention period $(P=0.236$; Figure $1 \mathrm{~A})$. When comparing the percentage of ideal stools
Table 1. Demographics and characteristics of the participants. ${ }^{1}$

$\begin{array}{ll}\text { Enrolled subjects, } \mathrm{n} & 135 \\ \text { Drop-out rate, } \mathrm{n}(\%) & 91(67.4) \\ \text { Mortality, } \mathrm{P}_{1} / \mathrm{P}_{2}(\%) & 4.4 / 2.3 \\ \text { Per protocol subjects, } \mathrm{n} & 44 \\ \text { Male/female, } \mathrm{n} / \mathrm{n} & 11 / 33 \\ \text { Age, mean } \pm \mathrm{SD}, \mathrm{y} & 83.8 \pm 5.9 \\ \text { Laxative usage }(\%) & \\ \text { Lactulose } & 9.6 \\ \text { MgO } & 7.0 \\ \text { Movicolon } & 49.0 \\ \text { Norgalax } & 4.2 \\ \text { Plum compote } & 29.0 \\ \text { Others } & 1.0\end{array}$

${ }^{1}$ Abbreviations used: $P_{1}=$ baseline period; $P_{2}=$ intervention period; SD = standard deviation; $\mathrm{MgO}$ = magnesium-oxide; Others = other laxatives are enema and bisacodyl.

(type 3 and 4) during the baseline- and intervention period, $36(\sim 82 \%)$ participants experienced an improvement in stool quality, whereas, for $8(\sim 18 \%)$ participants the stool quality worsened. The percentage of ideal stools per week during the intervention, as compared to baseline period was significantly higher $(61.4 \pm 28.6 \%$ vs $47.8 \pm 29.4 \%$; $P<0.01$; Figure $1 \mathrm{~B})$. When comparing the percentage of constipation-associated stool types (type 1 and 2) during the baseline- and intervention period, 21 ( 48\%) participants experienced an improvement, $16(\sim 36 \%)$ participants experienced no difference and for 7 ( 16\%) participants the stool quality worsened. Overall, there was a significantly lower percentage of constipation stool types per week during intervention, compared to baseline $(5.4 \pm 13.8 \%$ vs $13.2 \pm 23.5 \% ; P<0.01$; Figure $1 C$ ). When comparing the percentage of diarrhoea-associated stool types (type 5 , 6 and 7) during the baseline- and intervention period, $26(\sim 59 \%)$ participants experienced an improvement, 1 ( 2\%) participant experienced no difference and for 17 ( $39 \%)$ participants the stool quality worsened. Overall, there was a significant lower percentage of diarrhoea stool types per week during intervention, compared to baseline (33.2 $\pm 27.7 \%$ vs $39.0 \pm 31.6 \%$; $P<0.05$; Figure 1D).

Consumption of the probiotic product did not lower the number of patients obeying Rome III criterion $(\leq 2$ defecations per week) during the intervention period compared to the baseline $(P>0.05)$. The study product was well tolerated in all the participants. There occurred no adverse events (AEs) related to the study product during the trial. 

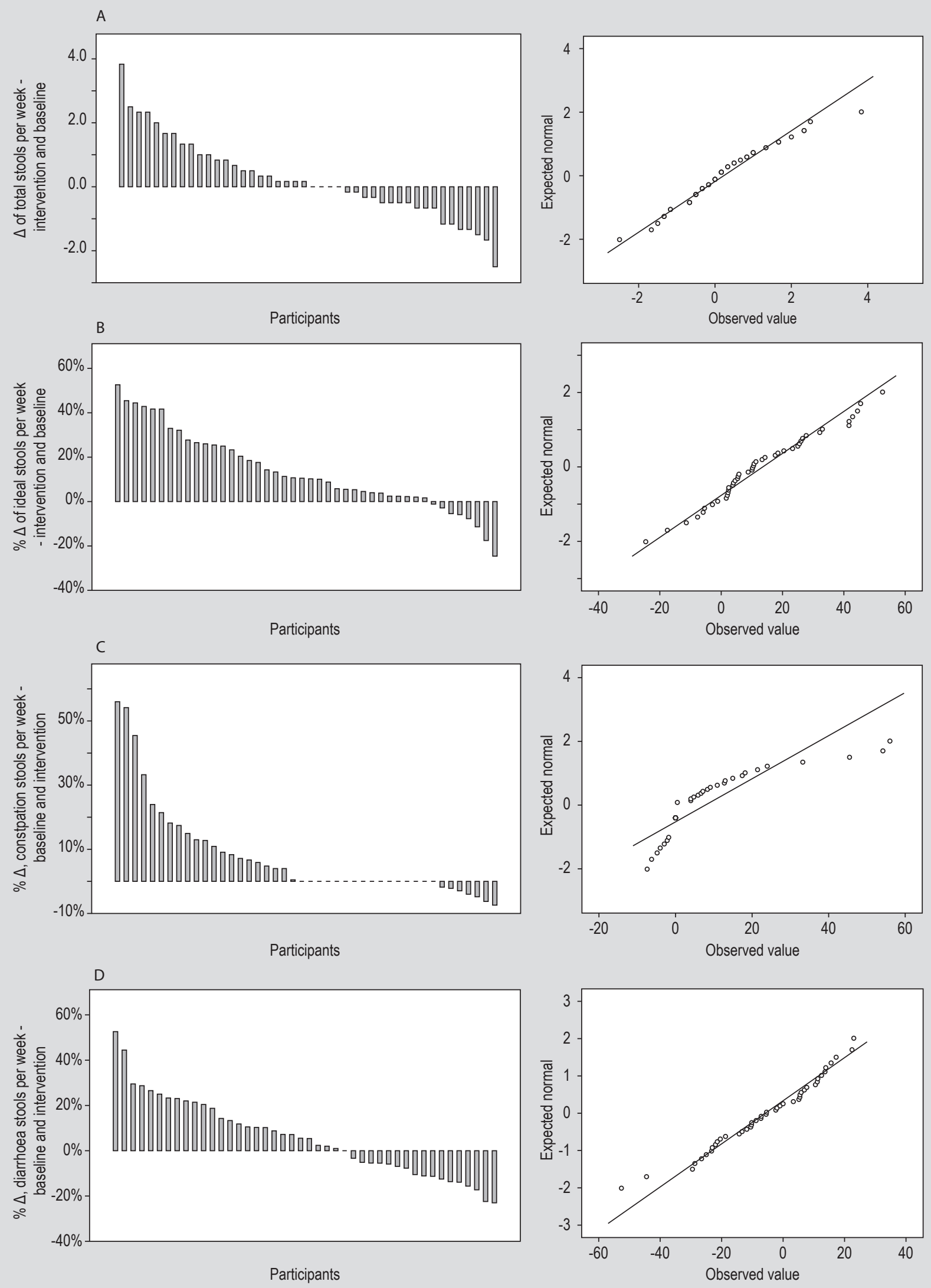

Figure 1. (A) Bowel movements, (B) ideal stool, (C) constipation and (D) diarrhoea. The displays show the improvements for each patient in percent points regarding the incidence of specified stool types per week (ideal/constipation/diarrhoea) between the intervention and baseline period respectively. Each figure is accompanied by its respective Q-Q-plot as a check for normality. C is not normally distributed. 


\section{Discussion}

The data of this pilot study indicate that $L c S$ fermented milk can significantly improve bowel habits of frail elderly residents in a nursing home. The use of a probiotic drink containing $L c S$ is already associated with boosting the immunity of elderly volunteers, for instance, by increasing the natural killer cell activity and displaying a more antiinflammatory cytokine profile (Dong et al., 2013). This suggests that probiotics can induce in addition to a local gastrointestinal effect, a far more widespread beneficial systemic effect.

The percentage of diarrhoea-associated stools (type 5, 6 and 7) per week was significantly lower during the probiotic intervention as compared to the baseline period. This might suggest that administration of a probiotic fermented milk beverage can reduce the incidence of diarrhoea in elderly residents in a nursing home. Indeed in children ageing 1-5 y, administration of $L c S$ had a protective effect of $14 \%$ (95\% CI: 4-23, $P<0.01$ ) for diarrhoea (Sur et al., 2011). Whether the stool types 5, 6 and 7 in the elderly were caused by AAD remains to be determined; stool types were not stratified for antibiotics usage. Wright et al. (in press), were not able to demonstrate an effect of a $L c S$-containing beverage on AAD in geriatric patients. However, this study was underpowered to detect a significant effect and the administration period was relatively short (Wright et al., in press). $L c S$ supplementation did show promising results in lowering the incidence of AAD ( 17.1 vs $54.9 \%, P<0.001)$ in patients with spinal cord injuries compared to the control group (Wong et al., 2014).

The percentage of constipation-associated stools (type 1 and 2) per week was significantly lower during the probiotic intervention compared to the baseline period. However, there was no significant difference in percentage of participants with $\leq 2$ defecations per week. Whether supplementation with $L c S$ improves stool consistency and defecation frequency in constipated participants remains questionably, as literature does not provide convincing evidence (Koebnick et al., 2003; Mazlyn et al., 2013; Sakai et al., 2011). If this is due to a relatively short intervention period remains to be determined. It is not unlikely that improvements in defecation frequency will be observed only after long-term administration. The reduction of both diarrhoea and constipation associated stool types resulted in a significantly higher percentage of ideal stool type (type 3 and 4) per week during the probiotic intervention compared to the baseline period.

Although the total number of bowel movements did not differ significantly compared to the baseline, other studies did show a moderate effect. In a randomised placebocontrolled trial, administration of $L c S$ in healthy individuals with soft stools significantly improved the stool quality and decreased the defecation frequency (Matsumoto et al., 2010). In constipated female students, consumption of a synbiotic fermented milk beverage containing $L c S$ resulted in a significant increase in bowel movements compared to the placebo group (Shioiri et al., 2006). Whereas these studies solely evaluated relatively healthy individuals with soft stool types and individuals with constipation, this current study demonstrated an improvement of all stool types (both diarrhoea and constipation) in relatively frail elderly individuals (as mentioned in the results).

The observed results in bowel habits were not related to a difference in laxative usage between the intervention- and baseline-period. The laxative intake was not significantly different during the intervention period compared to the baseline, demonstrating that the observed improvement in bowel habits can indeed be attributed to the introduction of the probiotic product and cannot be ascribed to changes in laxative usage. However, a further stratification of the data on laxative usage is, in this study, not possible due to limited number of patients that could be included for the statistical analysis. Preferably, a reduction in laxative usage is observed during the intervention period. However, there were no predetermined criteria for prescribing laxatives. Therefore it is possible that nursing staff were inclined to administer laxatives (e.g. plum compote), even if not necessary.

During this pilot study, no AEs were reported. This is in line with a previous study of van Puyenbroeck et al. (2012) were 737 individuals aged $\geq 65$ y in nursing homes were randomly assigned to receive a $L c S\left(n=375 ; 1.3 \times 10^{10} \mathrm{cfu}\right.$ $L c S /$ day $)$ or a placebo $(\mathrm{n}=362)$ for 21 days. During this study there were no AEs reported associated with consumption of L. casei Shirota, further advocating the safety of this strain in relative fragile individuals (Van Puyenbroek et al., 2012). In addition, no AEs were reported in very old individuals (mean age of 83 y) consuming a high dosage of $4.0 \times 10^{10}$ cfu LcS/day (Nagata et al., 2011). Currently, there are no indications that probiotics are unsafe in a controlled setting, even in preterm infants and severely immune comprised adults (Van den Nieuwboer et al., 2014, 2015). Indeed, the FAO designated a GRAS (generally recognised as safe) status to $\operatorname{LcS}$ (GRN000429).

In short, these data indicate that a $L c S$ fermented milk beverage significantly improves stool quality of frail elderly residents of a nursing home by a reduction of diarrhoea and constipation stool types and an increase in ideal stool types. These promising results should be confirmed by a randomised, controlled, parallel study. Whereas other strains (e.g. LGG) already have a well-established evidencebase for diarrhoea (Floch et al., 2011), data to support the use of $L c S$ to improve bowel habits is still in development. If these data can be confirmed, there is a huge opportunity to significantly improve the QoL of elderly in nursing homes and reduce healthcare costs and workload. 


\section{Supplementary material}

Supplementary material can be found online at http:// dx.doi.org/10.3920/BM2014.0113.

Figure S1. Sequential trial lay-out.

Figure S2. Bristol stool scale.

\section{Acknowledgements}

We gratefully acknowledge all personnel of Talma Hof, Emmeloord, the Netherlands, for their commitment and support. In addition we would like to thank Egbert Jan Vierkant (Yakult Nederland) for seminal discussions on data analysis, and Lidwien Franke (Yakult Nederland) for logistic support and fruitful discussions. Finally, we are grateful for the active participation of the residents of Talma Hof in this study.

\section{Conflict of interest}

This study was supported by Yakult Nederland BV, Amstelveen, the Netherlands. OL is Science Manager at Yakult Nederland BV. The other authors declared no conflict of interests.

\section{References}

Barat, I., Andreasen, F. and Damsgaard, E.M.S., 2000. The consumption of drugs by 75-year-old individuals living in their own homes. European Journal of Clinical Pharmacology 56: 501-509.

Biagi, E., Candela, M., Fairweather-Tait, S., Franceschi, C. and Brigidi, P., 2012. Ageing of the human metaorganism: the microbial counterpart. Age 34: 247-267.

Chmielewska, A. and Szajewska, H., 2010. Systematic review of randomised controlled trials: probiotics for functional constipation. World Journal of Gastroenterology 16: 69.

Claassen, E., 2014. Cost-benefit relation of diet and probiotics in iatrogenic bowel irregularity (IBI). Frontiers in Pharmacology 5: 14.

Claesson, M.J., Jeffery, I.B., Conde, S., Power, S.E., O'Connor, E.M., Cusack, S., Harris, H.M.B., Coakley, M., Lakshminarayanan, B., O'Sullivan, O., Fitzgerald, G.F., Deane, J., O'Conner, M., Harnedy, N., O'Conner, K., O'Mahony, D., Van Sinderen, D., Wallace, M., Brennan, L., Stanton, C., Marchesi, J. R., Fitzgerald, A. P., Shanahan, F., Hill, C. and O'Toole, P.W., 2012. Gut microbiota composition correlates with diet and health in the elderly. Nature 488: 178-184.

Dong, H., Rowland, I., Thomas, L.V. and Yaqoob, P., 2013. Immunomodulatory effects of a probiotic drink containing Lactobacillus casei Shirota in healthy older volunteers. European Journal of Nutrition 52: 1853-1863.

Drossman, D.A., Corazziari, E., Delvaux, M., Spiller, R.C., Talley, N.J., Thompson, W.G., Whitehead, W.E. (eds.), 2006. Rome III: The functional gastrointestinal disorders, $3^{\text {rd }}$ edition. Degnon Associates, McLean, VA, USA.
Duncan, S.H. and Flint, H.J., 2013. Probiotics and prebiotics and health in ageing populations. Maturitas 75: 44-50.

Floch, M.H., Walker, W.A., Madsen, K., Sanders, M.E., Macfarlane, G.T., Flint, H.J., Dieleman, L.A., Ringel, Y., Guandalini, S., Kelly, C.P. and Brandt, L.J., 2011. Recommendations for probiotic use 2011 update. Journal of Clinical Gastroenterology 45: S168-S171.

Food and Agriculture Organization/World Health Organization (FAO/ WHO), 2001. Report of a joint FAO/WHO Expert consultation on evaluation of health and nutritional properties of probiotics in food including powder milk with live lactic acid bacteria. Córdoba, Argentina. October, 1-4, 2001. FAO, Rome, Italy. Available at: http:// tinyurl.com/8bccc3r.

Gandell, D., Straus, S.E., Bundookwala, M., Tsui, V. and Alibhai, S.M., 2013. Treatment of constipation in older people. Canadian Medical Association Journal 185: 663.

Hell, M., Bernhofer, C., Stalzer, P., Kern, J.M. and Claassen, E., 2013. Probiotics in Clostridium difficile infection: reviewing the need for a multistrain probiotic. Beneficial Microbes 4: 39-51.

Hempel, S., Newberry, S.J., Maher, A.R., Wang, Z., Miles, J.N., Shanman, R., Johnsen, B. and Shekelle, P.G., 2012. Probiotics for the prevention and treatment of antibiotic-associated diarrhea: a systematic review and meta-analysis. Journal of the American Medical Association 307: 1959-1969.

Hill, C., Guarner, F., Reid, G., Gibson, G.R., Merenstein, D.J., Pot, B., Morelli, L., Canani, R. B., Flint, H.J., Salminen, S., Calder, P.C. and Sanders, M.E., 2014. Expert consensus document: the international scientific association for probiotics and prebiotics consensus statement on the scope and appropriate use of the term probiotic. Nature Reviews Gastroenterology and Hepatology 11: 506-514.

Karlström, O., Fryklund, B., Tullus, K. and Burman, L.G., 1998. A prospective nationwide study of Clostridium difficile-associated diarrhea in Sweden. Clinical Infectious Diseases 26: 141-145.

Koebnick, C., Wagner, I., Leitzmann, P., Stern, U. and Zunft, H.F., 2003. Probiotic beverage containing Lactobacillus casei Shirota improves gastrointestinal symptoms in patients with chronic constipation. Canadian Journal of Gastroenterology 17: 655-660.

Magrone, T. and Jirillo, E., 2013. The interaction between gut microbiota and age-related changes in immune function and inflammation. Immunity and Ageing 10: 31.

Mariat, D., Firmesse, O., Levenez, F., Guimarăes, V.D., Sokol, H., Dore, J., Corthier, G. and Furet, J.P., 2009. The Firmicutes/Bacteroidetes ratio of the human microbiota changes with age. BMC microbiology 9: 123 .

Matsumoto, K., Takada, T., Shimizu, K., Moriyama, K., Kawakami, K., Hirano, K., Kajimoto, O. and Nomoto, K., 2010. Effects of a probiotic fermented milk beverage containing Lactobacillus casei strain Shirota on defecation frequency, intestinal microbiota, and the intestinal environment of healthy individuals with soft stools. Journal of Bioscience and Bioengineering 110: 547-552.

Mazlyn, M.M., Nagarajah, L.H.L., Fatimah, A., Norimah, A.K. and Goh, K.L., 2013. Effects of a probiotic fermented milk on functional constipation: a randomized, double blind, placebo controlled study. Journal of Gastroenterology and Hepatology 28: 1141-1147. 
Mueller, S., Saunier, K., Hanisch, C., Norin, E., Alm, L., Midtvedt, T., Cresci, A., Silvi, S., Orpianesi, C., Verdenelli, M.C., Clavel, T., Koebnick, C., Zunft, H.J.F., Doré, J. and Blaut, M., 2006. Differences in fecal microbiota in different European study populations in relation to age, gender, and country: a cross-sectional study. Applied and Environmental Microbiology 72: 1027-1033.

Nagata, S., Asahara, T., Ohta, T., Yamada, T., Kondo, S., Bian, L., Wang, C., Yamashiro, Y. and Nomoto, K., 2011. Effect of the continuous intake of probiotic-fermented milk containing Lactobacillus casei strain Shirota on fever in a mass outbreak of norovirus gastroenteritis and the faecal microflora in a health service facility for the aged. British Journal of Nutrition 106: 549-556.

Rao, S.S. and Go, J.T., 2010. Update on the management of constipation in the elderly: new treatment options. Clinical Interventions in Aging 5: 163 .

Sakai, T., Makino, H., Ishikawa, E., Oishi, K. and Kushiro, A., 2011. Fermented milk containing Lactobacillus casei strain Shirota reduces incidence of hard or lumpy stools in healthy population. International Journal of Food Sciences and Nutrition 62: 423-430.

Sekirov, I., Russell, S.L., Antunes, L.C.M. and Finlay, B.B., 2010. Gut microbiota in health and disease. Physiological Reviews 90: 859-904.

Shioiri, T., Yahagi, K., Nakayama, S., Asahara, T., Yuki, N., Kawakami, K., Yamaoka, Y., Sakai, Y., Nomoto, K. and Totani, M., 2006. The effects of a synbiotic fermented milk beverage containing Lactobacillus casei strain Shirota and transgalactosylated oligosaccharides on defecation frequency, intestinal microflora, organic acid concentrations, and putrefactive metabolites of suboptimal health state volunteers: a randomized placebo-controlled cross-over study. Bioscience and Microflora 25: 137-146.

Sur, D., Manna, B., Niyogi, S. K., Ramamurthy, T., Palit, A., Nomoto, K., Takahashi, T., Shima, T., Tsuji, H., Kurakawa, T., Takeda, Y., Nair, G.B. and Bhattacharya, S.K., 2011. Role of probiotic in preventing acute diarrhoea in children: a community-based, randomized, double-blind placebo-controlled field trial in an urban slum. Epidemiology and Infection 139: 919-926.
Van den Nieuwboer, M., Brummer, R.J., Guarner, F., Morelli, L., Cabana, M. and Claassen, E., 2015. The administration of probiotics and synbiotics in immune compromised adults: is it safe? Beneficial Microbes 6: 3-17.

Van den Nieuwboer, M., Claassen, E., Morelli, L., Guarner, F. and Brummer, R.J., 2014. Probiotic and synbiotic safety in infants under two years of age. Beneficial Microbes 5: 45-60.

Van Dessel, C., Flamaing, J. and Hiele, M., 2005. Acute infectious (not Clostridium difficile-associated) diarrhea in the elderly. Tijdschrift voor Gerontologie en Geriatrie 36: 209-212.

Van Puyenbroeck, K., Hens, N., Coenen, S., Michiels, B., Beunckens, C., Molenberghs, G., Van Royen, P. and Verhoeven, V., 2012. Efficacy of daily intake of Lactobacillus casei Shirota on respiratory symptoms and influenza vaccination immune response: a randomized, doubleblind, placebo-controlled trial in healthy elderly nursing home residents. American Journal of Clinical Nutrition 95: 1165-1171.

Van Tongeren, S.P., Slaets, J.P., Harmsen, H.J.M. and Welling, G.W., 2005. Fecal microbiota composition and frailty. Applied and Environmental Microbiology 71: 6438-6442.

Videlock, E.J. and Cremonini, F., 2012. Meta-analysis: probiotics in antibiotic-associated diarrhoea. Alimentary Pharmacology \& Therapeutics 35: 1355-1369.

Wong, S., Jamous, A., O’Driscoll, J., Sekhar, R., Weldon, M., Yau, C.Y., Hirani, S.P., Grimble, G. and Forbes, A., 2014. A Lactobacillus casei Shirota probiotic drink reduces antibiotic-associated diarrhoea in patients with spinal cord injuries: a randomised controlled trial. British Journal of Nutrition 111: 672-678.

Wright, K., Wright, H. and Murray, M., in press. Probiotic treatment for the prevention of antibiotic-associated diarrhoea in geriatric patients: a multicentre randomised controlled pilot study. Australasian Journal on Ageing. DOI: http://dx.doi.org/10.1111/ ajag.12116. 
